\title{
Modelación del proceso precipitación-escurrimiento mensual por medio de regresiones
}

\author{
Modeling of the Monthly Rainfall-Runoff Process Through Regressions
}

\author{
Campos-Aranda Daniel Francisco \\ Profesor Jubilado de la Universidad Autónoma de San Luis Potosí \\ Correo:campos_aranda@hotmail.com
}

Información del artículo: recibido: julio de 2013, reevaluado: julio de 2013, aceptado: septiembre de 2013

\begin{abstract}
Resumen
Para la solución de los problemas asociados con la evaluación de los recursos hídricos de un río, la modelación del proceso precipitación-escurrimiento (PPE) permite deducir datos faltantes de escurrimiento, así como ampliar su registro, ya que por lo general la información disponible de precipitación tiene más amplitud. También hace posible la estimación de las entradas a embalses, cuando la construcción de estos suspendió a la estación de aforos. El modelo matemático más simple que se puede establecer para el PPE es la regresión lineal o curva, a nivel mensual. Este modelo se describe con detalle y se calibra con el registro conjunto de precipitación y escurrimiento mensuales de la estación hidrométrica Ballesmi, el cual abarca 35 años. Como el escurrimiento de la estación tiene una contribución importante de la descarga de manantiales, primero se corrigió su registro, retirando esa aportación. Para ello se desarrolló un procedimiento basado en los coeficientes de escurrimiento promedio mensual regionales o de una cuenca cercana y similar; en este caso se utilizó la estación de aforos Tancuilín. Ambas estaciones pertenecen a la Región Hidrológica Núm. 26 Parcial (Bajo Río Pánuco) y se ubican dentro del estado de San Luis Potosí. Los contrastes realizados indican que el modelo de regresión mensual, debido a su planteamiento conceptual, reproduce fielmente los volúmenes escurridos promedio mensual y alcanza una excelente aproximación en relación con su dispersión, lo anterior comprobado con base en las medias y las desviaciones estándar.
\end{abstract}

\section{Descriptores:}

- regresión lineal

- regresión polinomial

- coeficiente de correlación

- homogeneidad

- error relativo

- parámetros estadísticos 


\begin{abstract}
To solve the problems associated with the assessment of water resources of a river, the modeling of the rainfall-runoff process (RRP) allows the deduction of runoff missing data and to extend its record, since generally the information available on precipitation is larger. It also enables the estimation of inputs to reservoirs, when their building led to the suppression of the gauging station. The simplest mathematical model that can be set for the RRP is the linear regression or curve on a monthly basis. Such a model is described in detail and is calibrated with the simultaneous record of monthly rainfall and runoff in Ballesmi hydrometric station, which covers 35 years. Since the runoff of this station has an important contribution from the spring discharge, the record is corrected first by removing that contribution. In order to do this a procedure was developed based either on the monthly average regional runoff coefficients or on nearby and similar watershed; in this case the Tancuilin gauging station was used. Both stations belong to the Partial Hydrologic Region No. 26 (Lower Rio Panuco) and are located within the state of San Luis Potosi, México. The study performed indicates that the monthly regression model, due to its conceptual approach, faithfully reproduces monthly average runoff volumes and achieves an excellent approximation in relation to the dispersion, proved by calculation of the means and standard deviations.
\end{abstract}

\section{Introducción}

La modelación del proceso precipitación-escurrimiento (PPE) es necesaria cuando se deben estimar los recursos hídricos de un río y para ello, es conveniente ampliar su registro. En este caso se puede establecer un modelo del PPE a nivel mensual. En otras situaciones, como en el pronóstico de crecientes o en la estimación del impacto de descargas contaminantes, resulta indispensable la modelación del PPE a nivel diario e incluso horario. Beven (2001) establece y destaca que la razón teórica principal por la cual se requiere modelar el PPE, se debe a la limitación real que se tiene para medir todos los procesos hidrológicos que ocurren en una cuenca al transformar la precipitación en el escurrimiento. También cita ejemplos prácticos y generales asociados a la escasez de datos, donde es imprescindible la modelación del PPE, como en las cuencas sin aforos, las cuales no tienen información hidrométrica, o bien, cuando se debe estimar el impacto en el futuro de los cambios hidrológicos inducidos o probables, entonces las mediciones no son posibles.

Según Beven (2001), una primera clasificación general de los variados enfoques de modelación del PPE, distingue entre modelos globales y distribuidos, los primeros consideran a la cuenca como una unidad y sus variables y parámetros corresponden a los valores promedio de tal área; los segundos dividen la cuenca en áreas elementales y sus variables y parámetros se aso- cian a cada uno de tales elementos. La segunda diferencia básica es entre modelos determinísticos y estocásticos, los primeros producen una respuesta única para cada simulación que utiliza una serie de datos entrada y parámetros; en cambio los segundos permiten cierta aleatoriedad o incertidumbre en sus respuestas, debido a la variabilidad de sus variables de entrada, parámetros o condiciones de frontera. Otras clasificaciones, más detalladas o exhaustivas de los modelos del PPE se pueden consultar en Haan et al. (1982) y Singh (1995).

Desde que se inició la modelación del PPE hacia mediados de los años sesenta, los modelos conceptuales y los de caja negra son los que más han proliferado. Los primeros emplean representaciones matemáticas simplificadas de los principales procesos hidrológicos que ocurren en la cuenca y los segundos son totalmente matemáticos, se basan únicamente en las mediciones de entrada y salida de la cuenca, sin tomar en cuenta los procesos internos que transforman la precipitación en escurrimiento (Jones, 1997; Shaw et al., 2011). Los modelos que se emplean en este trabajo son del segundo tipo.

El objetivo de este trabajo consiste en describir con detalle el modelo general de regresión polinomial con memoria mensual, que considera a la precipitación como entrada y al escurrimiento como salida o respuesta. El modelo se aplica, para su calibración, a la cuenca de la estación hidrométrica Ballesmi del Río Coy de la Región Hidrológica Núm. 26 Parcial (Bajo Río Pánuco), 
en el estado de San Luis Potosí, que cuenta con un registro conjunto de precipitación y escurrimiento mensuales de 35 años. La estación de aforos Ballesmi fue seleccionada para este estudio, por tener un área de drenaje pequeña $\left(194 \mathrm{~km}^{2}\right)$ y disponer de tres estaciones pluviométricas, dos de ellas en su cuenca. Debido a que el escurrimiento de esta estación tiene una aportación importante de manantiales, primero se corrige con base en los coeficientes de escurrimiento promedio mensual observados en la estación de aforos Tancuilín, que también pertenece a la región hidrológica citada, es la más cercana, del mismo orden de magnitud $\left(321 \mathrm{~km}^{2}\right.$ ) y cuyo escurrimiento no está afectado por aportaciones subterráneas (IMTA, 2002). Los contrastes realizados muestran que los modelos de regresión mensuales permiten una buena aproximación para la estimación del escurrimiento mensual, ya que reproducen fielmente los valores promedio mensuales y se aproximan bastante a la dispersión observada.

\section{Resumen de la teoría operativa}

Modelo general de regresión

Propuesto y aplicado por Mimikou y Rao (1983) es el modelo matemático más simple que puede plantearse para reproducir el PPE a nivel mensual, sea este lineal o curvo. Su expresión general es:

$V_{i, j}=\sum_{k=0}^{K} \beta_{j, k} \cdot\left(\frac{P_{i, j}+P_{i, j-1}+\cdots+P_{i, j-n}}{n+1}\right)^{k}$

siendo, $V_{i, j}$ el escurrimiento mensual del $i$-ésimo año, con $i$ variando de 1 a $N$, que es la amplitud del registro conjunto procesado y $j$ es número de mes, con 1 para enero y 12 para diciembre. Es común expresar a $V_{i, j}$ en la ecuación 1, en milímetros, lo cual equivale a dividir su valor en miles de metros cúbicos $\left(10^{3} \mathrm{~m}^{3}\right)$ entre el área de cuenca $A$ en $\mathrm{km}^{2}$. $P_{i, j}$ es la precipitación mensual representativa de la cuenca en milímetros. $K$ y $n$ son los parámetros del modelo y $\beta_{j, k}$ son los coeficientes de regresión del modelo, los cuales se obtienen mediante un ajuste de mínimos cuadrados de los residuos.

\section{Selección de los parámetros del modelo}

Los parámetros $K$ y $n$ definen la estructura del modelo. El primero determina su orden como ecuación de regresión polinomial y el segundo su memoria con respecto a la precipitación mensual. Entonces, para estimar el valor de $K$ se debe establecer la relación funcional entre el escurrimiento y la precipitación de cada mes, el primero como la variable dependiente en las ordenadas y la segunda como la variable independiente en las abscisas. Como esta relación puede ser lineal o curva, en el primer caso $K=1$ y en el segundo $K>1$. No es común encontrar valores de $K$ diferentes en cada mes $y$, por ello, este parámetro es considerado una característica de la cuenca (Mimikou y Rao, 1983).

Cuando alguna relación funcional mensual muestre gran dispersión, se debe establecer la relación entre $V_{i, j}$ y un valor promedio de las precipitación de los meses $j$, $j-1, \ldots, j-n$, con $n$ indicando la memoria del proceso en el mes $j$. Por lo común, con $n=1$ es suficiente para mejorar la relación, en cuencas pequeñas.

Coeficientes de regresión y correlación de la relación funcional

Cuando $K=1$ la ecuación 1 es una línea recta, con $\beta_{j, 0}$ como ordenada al origen y $\beta_{j, 1}$ como pendiente de la recta, cuyas expresiones del ajuste por mínimos cuadrados de los residuos son (Campos, 2003):

$\beta_{j, 1}=\frac{\sum_{i=1}^{N} P_{i, j} \cdot V_{i, j}-N \cdot \bar{P}_{j} \cdot \bar{V}_{j}}{\sum_{i=1}^{N}\left(P_{i, j}\right)^{2}-N \cdot\left(\bar{P}_{j}\right)^{2}}$

$\beta_{j, 0}=\bar{V}_{j}-\beta_{j, 1} \cdot \bar{P}_{j}$

En las ecuaciones anteriores, $P_{i, j}$ es la precipitación mensual cuando $n=0$ y puede ser el promedio de la de ese mes y el anterior, si $n=1 . \bar{P}_{j}$ y $\bar{V}_{j}$ son los valores promedio mensual de la precipitación y el escurrimiento, ambos en milímetros. La medida cuantitativa de la relación funcional entre la precipitación y el escurrimiento, se obtiene a través del coeficiente de correlación lineal $\left(R_{x y}^{j}\right)$, que establece el grado de asociación o dependencia entre ambas variables, con cero para puntos totalmente dispersos y uno cuando todos están sobre la línea recta. Su expresión es:

$R_{x y}^{j}=\frac{\sum_{i=1}^{N}\left(P_{i, j}-\bar{P}_{j}\right) \cdot\left(V_{i, j}-\bar{V}_{j}\right)}{\left[\sum_{i=1}^{N}\left(P_{i, j}-\bar{P}_{j}\right)^{2} \cdot \sum_{i=1}^{N}\left(V_{i, j}-\bar{V}_{j}\right)^{2}\right]^{1 / 2}}$

Cuando la relación funcional entre la precipitación y el escurrimiento es curva $(K>1)$ se deberá ajustar un modelo de regresión polinomial, parabólico $(K=2)$ o cúbico 
$(K=3)$, cuya solución se obtiene con un planteamiento matricial que se puede consultar en Campos (2003), así como su coeficiente de determinación $\left(R^{2}\right)$, el cual indica qué proporción de la variabilidad de la variable dependiente es explicada por la regresión polinomial.

\section{Información hidrométrica procesada}

Estación de aforos Ballesmi

Perteneciente a la Región Hidrológica Núm. 26 Parcial (Bajo Río Pánuco) fue seleccionada para este estudio por ser una cuenca pequeña que tiene dos estaciones pluviométricas dentro de ella, Santa Cruz y Aquismón, además de la ubicada en el sitio de tal hidrométrica. Mide los escurrimientos del río Coy afluente del Río Tampaón, su área de cuenca es de $194 \mathrm{~km}^{2}$ y su precipitación media anual se estima en los 2000 milímetros (INEGI, 1980). Su registro de volúmenes escurridos mensuales comenzó en noviembre de 1953 y se tiene disponible hasta diciembre de 2002 en el sistema BANDAS (IMTA, 2002), bajo la clave 26241. En el lapso citado, el año de 1979 no tiene datos y los años de 1991,
1998 y 2000 están incompletos. La descripción de su régimen de escurrimiento establece que esta corriente recibe la aportación de los manantiales de El Nacimiento, por ello, primero se debe estimar esa componente subterránea, para eliminarla y después intentar modelar el PPE de manera independiente.

En la figura 1 se muestra la cuenca de la estación Ballesmi y sus respectivas estaciones pluviométricas, cuyos registros de precipitación mensual iniciaron en enero de 1961 y están disponibles hasta diciembre de 2012. Tales registros fueron proporcionados por la Dirección Estatal San Luis Potosí de la CONAGUA. Entonces el registro conjunto de escurrimiento y precipitación mensuales abarcó de enero de 1961 a diciembre de 1997, con dos años faltantes: 1979 y 1991, es decir 35 años.

Todos los valores mensuales faltantes de precipitación de la cuenca de la estación de aforos Ballesmi, fueron adoptados igual a la moda de tal mes, estimada con base en la distribución Gamma mixta (Campos, 2005), utilizando todo el registro mensual disponible, por ello variaron de 48 a 52 datos. Cuando la moda no existe se emplea la precipitación confiable $(P C)$, cuya probabili-

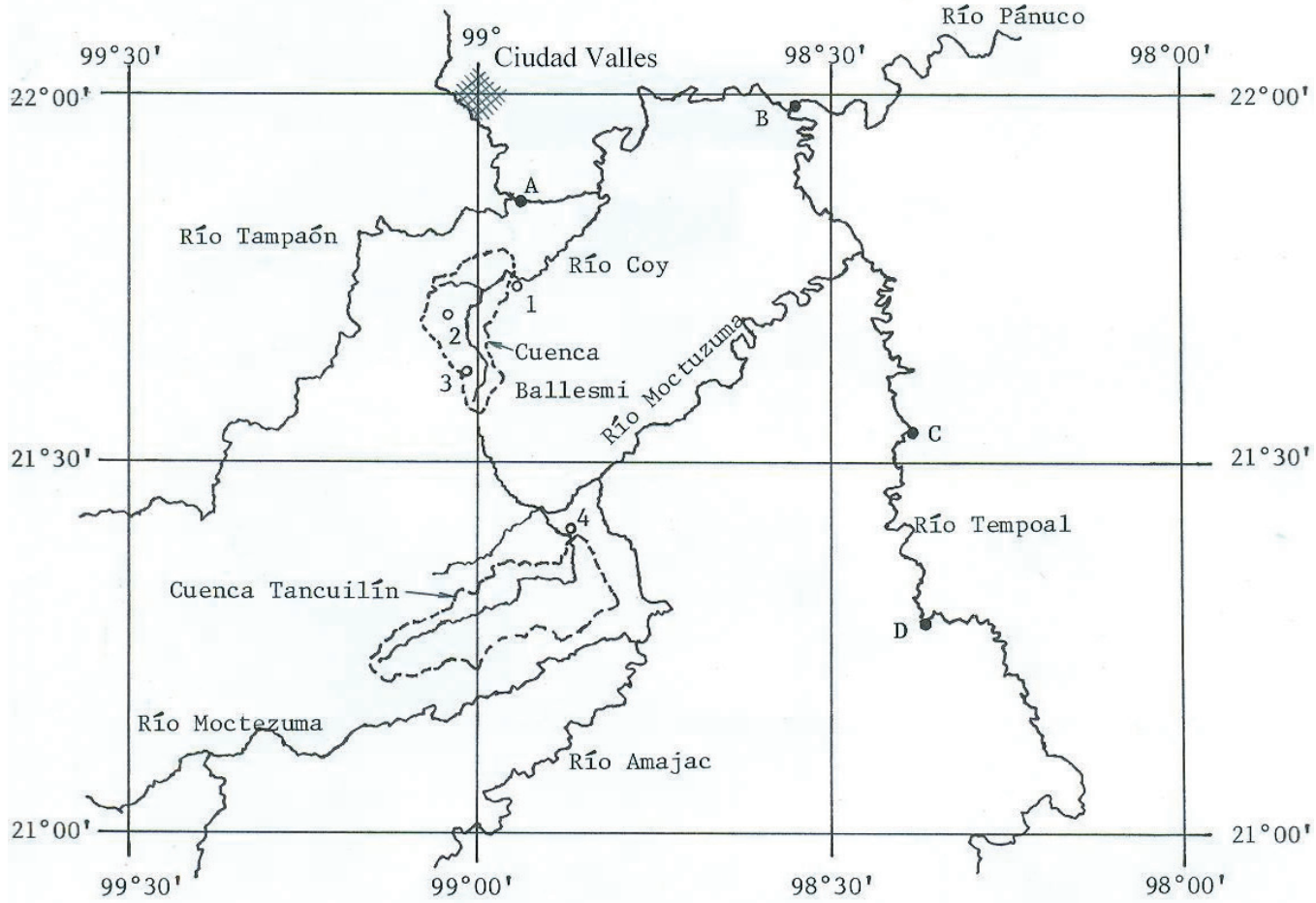

Estaciones de aforos:

$$
\begin{array}{ll}
\text { A } & \text { El Pujal } \\
\text { B } & \text { Las Adjuntas } \\
\text { C } & \text { Tempoal } \\
\text { D } & \text { Platón Sánchez }
\end{array}
$$

Estaciones pluviométricas:

$$
\begin{array}{ll}
1 & \text { Ballesmi } \\
2 & \text { Santa Cruz } \\
3 & \text { Aquismón } \\
4 & \text { Tancuilín }
\end{array}
$$

Figura 1. Localización de las cuencas y pluviómetros de las estaciones hidrométricas Ballesmi y Tancuilín en la Región Hidrológica Núm. 26 Parcial (Bajo Río Pánuco) 
dad de excedencia es de $75 \%$, es decir, que en cada cuatro años solo uno tiene una precipitación menor. En la tabla 1 se citan los resultados de los análisis mensuales, únicamente para la estación pluviométrica Ballesmi.

\section{Estación de aforos Tancuilín}

También ubicada dentro de la misma región hidrológica, es la más cercana a la cuenca de la estación Ballesmi, con régimen de escurrimiento no afectado por manantiales (IMTA, 2002) y con área de cuenca reducida. Sus datos generales se han concentrado en la tabla 2. La estación hidrométrica Tancuilín solo tiene la estación pluviométrica del mismo nombre ubicada en su sitio. En la figura 1 se muestra su cuenca.

Se observa en la tabla 2 que el VEMA de la estación de aforos Ballesmi es más del doble del medido en la estación Tancuilín; la cual tiene un área de cuenca más grande un $65 \%$ y también mayor Pmac. Lo anterior pone de manifiesto la importancia de la aportación de manantiales en la estación Ballesmi.

Verificación de la homogeneidad de los registros

Antes de realizar alguna estimación estadística con un registro mensual de volumen escurrido o de precipitación, se debe verificar su homogeneidad, lo cual signifi- ca que pertenezca a una sola población y que esté libre de componentes determinísticas. Para probar lo anterior, se aplicaron a los registros anuales de escurrimiento y precipitación, siete pruebas (WMO, 1971; Buishand, 1982 y Campos, 2005). La de Von Neumann (VN) que detecta falta de aleatoriedad contra componentes determinísticas no especificadas, dos de persistencia, la de Anderson (PA) y la de Sneyers (PS) y dos de tendencia, la de Kendall (TK) y la de Spearman (TS). La prueba de Cramer (PC) relativa a cambios en la media, se aplicó utilizando dos subperiodos iguales a la mitad del registro, uno al inicio y otro al final. Por último, la prueba de constancia de variabilidad de Bartlett (VB) se aplicó dividiendo cada registro en tres subperiodos.

En la tabla 3 se han concentrado los registros conjuntos anuales de la estación de aforos Ballesmi y de los tres pluviómetros de su cuenca y en la tabla 4 se tienen los resultados de las siete pruebas citadas. Se observa que únicamente tres registros de la estación hidrométrica Ballesmi presentan ligera persistencia detectada con la prueba de Spearman.

Tabla 1. Resultados del ajuste mensual de la distribución Gamma Mixta a los datos de la estación pluviométrica Ballesmi

\begin{tabular}{lcccccccccccc}
\hline \multicolumn{1}{c}{ Concepto } & $\mathrm{E}$ & $\mathrm{F}$ & $\mathrm{M}$ & $\mathrm{A}$ & $\mathrm{M}^{\prime}$ & $\mathrm{J}$ & $\mathrm{J}^{\prime}$ & $\mathrm{A}^{\prime}$ & $\mathrm{S}$ & $\mathrm{O}$ & $\mathrm{N}$ & $\mathrm{D}$ \\
\hline Núm. de datos & 50 & 51 & 49 & 52 & 51 & 51 & 51 & 51 & 51 & 49 & 47 & 48 \\
Núm. de ceros & 0 & 0 & 1 & 2 & 1 & 0 & 0 & 0 & 0 & 0 & 0 & 1 \\
Mediana & 27.1 & 26.7 & 23.1 & 39.2 & 92.0 & 209.7 & 192.9 & 158.5 & 263.3 & 120.2 & 45.5 & 30.9 \\
Moda & 9.4 & 10.1 & 5.0 & $\mathrm{NE}^{*}$ & 50.9 & 126.5 & 35.0 & 80.4 & 188.2 & 63.2 & 21.5 & 11.0 \\
PC & 13.7 & 13.8 & 10.5 & 15.3 & 50.8 & 123.6 & 89.9 & 87.9 & 168.4 & 67.4 & 24.7 & 15.2 \\
\hline
\end{tabular}

* no existe, la distribución mensual es de tipo J invertida

Tabla 2. Características generales de las estaciones hidrométricas procesadas y de sus respectivas estaciones pluviométricas

\begin{tabular}{ccccccc}
\hline $\begin{array}{c}\text { Hidrométrica } \\
\text { Pluviométrica }\end{array}$ & Clave & Latitud N. & $\begin{array}{c}\text { Longitud } \\
\text { W.G. }\end{array}$ & $\begin{array}{c}A-\text { Pmac } \\
\text { (\% área) }\end{array}$ & $\begin{array}{c}\text { Periodo de registro } \\
\text { (número de años) }\end{array}$ & $\begin{array}{c}\text { VEMA } \\
\text { Pmab }\end{array}$ \\
\hline Ballesmi & 26241 & $21^{\circ} 45^{\prime}$ & $98^{\circ} 56^{\prime}$ & $194-2,000$ & $1961-1997(35)$ & 914682.0 \\
Ballesmi & 24005 & $21^{\circ} 45^{\prime}$ & $98^{\circ} 56^{\prime}$ & $(25)$ & $1961-1997(35)$ & 1491.5 \\
Santa Cruz & 24072 & $21^{\circ} 43^{\prime}$ & $99^{\circ} 03^{\prime}$ & $(45)$ & $1961-1997(35)$ & 1823.2 \\
Aquismón & 24003 & $21^{\circ} 38^{\prime}$ & $99^{\circ} 02^{\prime}$ & $(30)$ & $1961-1997(35)$ & 2257.6 \\
Tancuilín & 26291 & $21^{\circ} 23^{\prime}$ & $98^{\circ} 52^{\prime}$ & $321-2,950$ & $1961-1994(34)$ & 453445.0 \\
Tancuilín & 24084 & $21^{\circ} 23^{\prime}$ & $98^{\circ} 52^{\prime}$ & $(100)$ & $1961-1994(34)$ & 2240.9 \\
\hline
\end{tabular}

Simbología:

A $\quad=$ área de cuenca, en $\mathrm{km}^{2}$

Pmac = precipitación media anual en la cuenca, en milímetros

$\%$ área $\quad=$ porcentaje de área de cuenca asignado a tal pluviómetro

VEMA = volumen escurrido medio anual, en miles de $\mathrm{m}^{3}$

Pmab = precipitación media anual en la estación base, en milímetros 


\section{Estimación de la Aportación de Manantiales}

El procedimiento que se propone para la estimación de la aportación subterránea en el escurrimiento total observado en la estación de aforos Ballesmi obedece al hecho de no contar con información hidrométrica relativa a las aportaciones de los manantiales y por ello se basa en el comportamiento hidrológico observado en una cuenca cercana, de tamaño similar y que se ubica dentro de la misma subregión geográfica.

Específicamente, el procedimiento sugerido acepta dos suposiciones básicas. La primera consiste en utilizar los coeficientes de escurrimiento promedio men- suales $\left(C e_{j}\right)$ observados en la estación hidrométrica Tancuilín, para estimar el escurrimiento directo en Ballesmi, función de su área de cuenca y de su precipitación respectiva. En relación con esta consideración, se ha encontrado que los $C e_{j}$ muestran similitud en cuencas que pertenecen a la misma subregión geográfica (Campos, 2013). La segunda suposición admite que la aportación de manantiales también varía con la precipitación y entonces cada escurrimiento mensual observado será corregido por una aportación subterránea modificada, con base en su error relativo respecto al promedio mensual observado. El procedimiento sugerido se desarrolla en los 4 pasos siguientes.

Tabla 3. Valores anuales del escurrimiento $\left(10^{3} \cdot \mathrm{m}^{3}\right)$ y la precipitación $(\mathrm{mm})$ en las estaciones de la cuenca de la estación de aforos Ballesmi

\begin{tabular}{rrrrrrrrrc}
\hline \multicolumn{1}{c}{ Precipitación anual en } & \multicolumn{3}{c}{ Precipitación anual en } \\
Año & \multicolumn{1}{c}{ VEAO } & Ballesmi & S. Cruz & Aquismón & Año & VEAO & Ballesmi & S. Cruz & Aquismón \\
\hline 1961 & 875411 & 1176.1 & 1512.9 & 2075.0 & 1980 & 731731 & 1100.8 & 1504.0 & 1768.2 \\
1962 & 695256 & 823.8 & 1223.0 & 1248.5 & 1981 & 1106292 & 1857.0 & 2515.1 & 2950.4 \\
1963 & 667781 & 1089.5 & 1296.5 & 959.2 & 1982 & 579843 & 1017.1 & 1117.8 & 1480.4 \\
1964 & 678010 & 1005.7 & 1396.1 & 1607.2 & 1983 & 858750 & 1395.2 & 1771.8 & 2175.4 \\
1965 & 799757 & 1413.9 & 1858.5 & 2196.6 & 1984 & 1127368 & 1847.2 & 2222.2 & 3168.9 \\
1966 & 1014229 & 1668.4 & 1735.0 & 3148.4 & 1985 & 874027 & 1399.8 & 2084.6 & 2533.7 \\
1967 & 1022135 & 1463.8 & 1989.0 & 2162.2 & 1986 & 813209 & 1253.3 & 1835.0 & 1776.2 \\
1968 & 956144 & 1623.9 & 2023.6 & 2634.4 & 1987 & 802588 & 929.0 & 1348.0 & 1628.5 \\
1969 & 1023727 & 1511.2 & 1515.2 & 2292.9 & 1988 & 944821 & 1455.2 & 1630.5 & 2142.5 \\
1970 & 1048881 & 1893.0 & 2388.8 & 2872.7 & 1989 & 811741 & 1211.7 & 1532.0 & 2210.0 \\
1971 & 967997 & 1638.9 & 2111.7 & 2123.0 & 1990 & 978538 & 1530.3 & 1304.1 & 2023.5 \\
1972 & 1015631 & 1566.0 & 2317.1 & 2974.9 & 1992 & 1071126 & 1963.9 & 2419.5 & 2607.0 \\
1973 & 1120103 & 2216.2 & 2444.1 & 2847.2 & 1993 & 1408598 & 2955.3 & 2535.3 & 3541.5 \\
1974 & 1053913 & 1771.8 & 2026.2 & 2369.0 & 1994 & 775327 & 1380.9 & 1562.2 & 1947.0 \\
1975 & 1094053 & 1673.4 & 2451.2 & 2172.7 & 1995 & 751937 & 1089.3 & 1578.6 & 2239.5 \\
1976 & 1208742 & 2360.6 & 2712.9 & 3562.1 & 1996 & 797767 & 1080.0 & 1860.5 & 1663.0 \\
1977 & 699825 & 1127.1 & 1169.6 & 1778.6 & 1997 & 679166 & 1111.1 & 1151.0 & 1647.5 \\
1978 & 959427 & 1603.2 & 1668.4 & 2488.3 & media & 914682 & 1491.5 & 1823.2 & 2257.6 \\
\hline
\end{tabular}

VEAO = volumen escurrido anual observado, en miles de $\mathrm{m}^{3}$

Tabla 4. Resultados de las pruebas estadísticas específicas aplicadas a los registros anuales de las estaciones hidrométricas Ballemi y Tancuilín y sus estaciones pluviométricas

\begin{tabular}{|c|c|c|c|c|c|c|c|c|c|c|c|c|}
\hline \multirow{2}{*}{$\begin{array}{c}\text { Hidrométrica } \\
\text { pluviométricas }\end{array}$} & \multicolumn{4}{|c|}{ Parámetros estadísticos } & \multicolumn{8}{|c|}{ Pruebas estadísticas aplicadas } \\
\hline & S & $C v$ & Cs & $r_{1}$ & VN & PA & PS & TK & TS & $\mathrm{PC}_{1}$ & $\mathrm{PC}_{2}$ & VB \\
\hline Ballesmi & $18219310^{3} \cdot \mathrm{m}^{3}$ & 0.199 & 0.369 & 0.199 & $\mathrm{H}$ & $\mathrm{H}$ & $\mathrm{NH}$ & $\mathrm{H}$ & $\mathrm{H}$ & $\mathrm{H}$ & $\mathrm{H}$ & $\mathrm{H}$ \\
\hline Ballesmi & $443.5 \mathrm{~mm}$ & 0.297 & 1.197 & 0.241 & $\mathrm{H}$ & $\mathrm{H}$ & $\mathrm{NH}$ & $\mathrm{H}$ & $\mathrm{H}$ & $\mathrm{H}$ & $\mathrm{H}$ & $\mathrm{H}$ \\
\hline Santa Cruz & $459.2 \mathrm{~mm}$ & 0.252 & 0.239 & 0.166 & $\mathrm{H}$ & $\mathrm{H}$ & $\mathrm{NH}$ & $\mathrm{H}$ & $\mathrm{H}$ & $\mathrm{H}$ & $\mathrm{H}$ & $\mathrm{H}$ \\
\hline Aquismón & $616.0 \mathrm{~mm}$ & 0.273 & 0.271 & 0.122 & $\mathrm{H}$ & $\mathrm{H}$ & $\mathrm{H}$ & $\mathrm{H}$ & $\mathrm{H}$ & $\mathrm{H}$ & $\mathrm{H}$ & $\mathrm{H}$ \\
\hline Tancuilín & $20081310^{3} \cdot \mathrm{m}^{3}$ & 0.443 & 0.531 & -0.026 & $\mathrm{H}$ & $\mathrm{H}$ & $\mathrm{H}$ & $\mathrm{H}$ & $\mathrm{H}$ & $\mathrm{H}$ & $\mathrm{H}$ & $\mathrm{H}$ \\
\hline Tancuilín & $2240.9 \mathrm{~mm}$ & 0.232 & -0.072 & 0.092 & $\mathrm{H}$ & $\mathrm{H}$ & $\mathrm{H}$ & $\mathrm{H}$ & $\mathrm{H}$ & $\mathrm{H}$ & $\mathrm{H}$ & $\mathrm{H}$ \\
\hline
\end{tabular}

Simbología:

$S=$ desviación estándar

$C V=$ coeficiente de variación

Cs = coeficiente de asimetría

$r_{1}=$ coeficiente de correlación serial de orden 1
$\mathrm{H}$ = homogénea

$\mathrm{NH}=$ no homogénea 
Paso 1) Estimación de los $\mathrm{Ce}_{j}$. Con base en los valores promedio mensuales de escurrimiento $\left(\bar{V}_{j}\right)$ y precipitación $\left(\overline{P b}_{j}\right)$ de la estación de aforos Tancuilín se estimarán sus coeficientes de escurrimiento promedio mensuales $\left(C e_{j}\right)$, con la expresión siguiente:

$$
C e_{j}=\frac{\bar{V}_{j}}{A \cdot F c \cdot \overline{P b}_{j}}
$$

donde $A$ es su área de cuenca en $\mathrm{km}^{2}$ y $F c$ es su factor de transporte o correctivo, definido como:

$$
F c=\frac{P m a c}{P m a b}
$$

En los tres primeros renglones de valores de la tabla 5 se tienen los datos y resultados de la aplicación de la ecuación 5, en la cual, $F c=1.3164$, según magnitudes de la tabla 2.

Paso 2) Precipitación representativa. De acuerdo con la ubicación de las estaciones pluviométricas en la cuenca, se obtienen sus factores de ponderación para obtener el registro integrado. En la cuenca de la estación de aforos Ballesmi, se estimaron valores de 25, 45 y 30\% para los pluviómetros de Ballesmi, Santa Cruz y Aquismón, respectivamente, según técnica de los polígonos de Thiessen. En el antepenúltimo renglón de valores de la tabla 5 , se exponen los promedios mensuales de precipitación del registro integrado.

Paso 3) Estimación de la Aportación Subterránea. Multiplicando los $C e_{j}$ de Tancuilín por el área de cuenca $(A$, en $\mathrm{km}^{2}$ ) de Ballesmi y por su precipitación mensual del registro integrado, corregida por $F_{C}=1.0692$, se obtienen las magnitudes estimadas para el volumen escurrido superficial $\left(\bar{V}_{s}\right)$, mostrados en el penúltimo renglón de la tabla 5. Lo anterior equivale a aplicar la ecuación 5, para obtener $\bar{V}_{j}$. Restando estos valores al escurrimiento total observado se obtienen las aportaciones subterráneas promedio mensuales $\left(A S P_{j}\right)$, expuestas en el último renglón de la tabla 5.

Paso 4) Corrección del escurrimiento mensual. Primeramente se obtiene el error relativo $(E R)$ de cada escurrimiento mensual observado respecto a su promedio mensual, su expresión es:

Tabla 5. Estimación de los volúmenes de escurrimiento directo y subterráneo en la estación de aforos Ballesmi, con base en los datos

\begin{tabular}{|c|c|c|c|c|c|c|c|c|c|c|c|c|c|}
\hline $\begin{array}{l}\text { Hidrométrica y } \\
\text { Concepto }\end{array}$ & E & $\mathrm{F}$ & $\mathrm{M}$ & A & $\mathrm{M}^{\prime}$ & $\mathrm{J}$ & $\mathrm{J}^{\prime}$ & $\mathrm{A}^{\prime}$ & S & $\mathrm{O}$ & $\mathrm{N}$ & $\mathrm{D}$ & Anual \\
\hline \multicolumn{14}{|l|}{$\begin{array}{l}\text { Tancuilín } \\
\left(\mathrm{A}=321 \mathrm{~km}^{2}\right)\end{array}$} \\
\hline $\begin{array}{l}\text { Esc. observado } \\
\left(10^{3} \mathrm{~m}^{3}\right)\end{array}$ & 6345 & 4841 & 4573 & 6458 & 12131 & 52447 & 72621 & 64739 & 127327 & 66605 & 23914 & 11444 & 453445 \\
\hline $\begin{array}{l}\text { Precip. representativa } \\
(\mathrm{mm})\end{array}$ & 72.5 & 61.9 & 66.8 & 84.8 & 189.5 & 299.6 & 313.8 & 295.9 & 448.1 & 215.8 & 121.6 & 70.6 & 2240.9 \\
\hline $\begin{array}{l}\text { Coeficientes de Esc. } \\
(\mathrm{Ce})\end{array}$ & 0.207 & 0.185 & 0.162 & 0.180 & 0.152 & 0.414 & 0.548 & 0.518 & 0.672 & 0.730 & 0.465 & 0.384 & 0.479 \\
\hline \multicolumn{14}{|l|}{$\begin{array}{l}\text { Ballesmi } \\
\left(\mathrm{A}=194 \mathrm{~km}^{2}\right)\end{array}$} \\
\hline $\begin{array}{l}\text { Esc. observado } \\
\left(10^{3} \mathrm{~m}^{3}\right)\end{array}$ & 48763 & 43547 & 46798 & 47769 & 56249 & 98121 & 113864 & 104217 & 141828 & 100565 & 59677 & 53284 & 914682 \\
\hline $\begin{array}{l}\text { Precip. en Ballesmi } \\
(\mathrm{mm})\end{array}$ & 36.5 & 36.0 & 32.2 & 56.7 & 129.0 & 263.4 & 216.0 & 197.8 & 298.7 & 131.6 & 55.0 & 38.6 & 1491.5 \\
\hline $\begin{array}{l}\text { Precip. en Santa Cruz } \\
(\mathrm{mm})\end{array}$ & 37.8 & 51.5 & 43.0 & 70.1 & 153.9 & 323.3 & 260.4 & 274.6 & 344.4 & 152.8 & 71.6 & 39.9 & 1823.2 \\
\hline $\begin{array}{l}\text { Precip. en Aquismón } \\
(\mathrm{mm})\end{array}$ & 63.4 & 67.2 & 75.7 & 107.5 & 185.0 & 364.6 & 292.8 & 311.0 & 440.8 & 189.7 & 96.2 & 63.4 & 2257.6 \\
\hline $\begin{array}{l}\text { Precip. Reg. integrado } \\
(\mathrm{mm})\end{array}$ & 45.2 & 52.3 & 50.1 & 78.0 & 157.0 & 320.7 & 259.0 & 266.3 & 361.9 & 158.6 & 74.8 & 46.6 & 1870.5 \\
\hline $\begin{array}{l}\text { Esc. Superficial estim. } \\
\left(10^{3} \mathrm{~m}^{3}\right)\end{array}$ & 1941 & 2007 & 1684 & 2912 & 4950 & 27540 & 29440 & 28613 & 50445 & 24015 & 7215 & 3712 & 184474 \\
\hline $\begin{array}{l}\text { Esc. Subterráneo } \\
\left(10^{3} \mathrm{~m}^{3}\right)\end{array}$ & 46822 & 41540 & 45114 & 44857 & 51299 & 70581 & 84424 & 75604 & 91383 & 76550 & 52462 & 49572 & 730208 \\
\hline
\end{tabular}
de la estación hidrométrica Tancuilín 
$E R_{i, j}=\frac{\left(V_{i, j}-\bar{V}_{j}\right)}{\bar{V}_{j}}$

Los valores del $E R_{i, j}$ serán positivos cuando el escurrimiento observado excede al promedio y negativos cuando ocurre lo contrario. Para obtener el volumen escurrido corregido mensual $\left(V c_{i, j}\right)$, al escurrimiento observado se le resta la aportación subterránea modificada por el $E R_{i, j^{\prime}}$ esto es:

$V c_{i, j}=V_{i, j}-\left(1+E R_{i, j}\right) \cdot A S P_{j}$

El procedimiento sugerido conduce a unos valores promedio mensuales de $V c_{i, j}$ denominados $\overline{V_{c}}$ iguales a los $\overline{V s}_{j}$, estimados previamente. Al aplicar el Paso 4, se obtiene el registro mensual de volumen escurrido corregido.

\section{Análisis de los resultados}

\section{Relaciones funcionales}

En las figuras 2 y 3 se muestran las relaciones funcionales de los meses de marzo y junio, del registro corregido. Se observa que son lineales al igual que las del resto de los meses. Por otra parte, en la tabla 6 se indican los coeficientes de correlación $\left(R_{x y}\right)$ obtenidos al realizar el ajuste lineal de mínimos cuadrados de los residuos (ecuación 4). En todos los ajustes no se eliminaron valores dispersos, por lo tanto el número de parejas $(N)$ siempre fue de 35 .
Los valores de la tabla 6 indican que en los meses de febrero, marzo, noviembre y diciembre se tienen los valores de $R_{x y}$ más bajos. Para definir el valor mínimo del $R_{x y}$ que es estadísticamente diferente de cero, se aplicó una prueba basada en la distribución $t$ de Student (Yevjevich, 1972) con un nivel $\alpha$ de significancia de $5 \%$, obteniéndose para $N=35$ un valor de $R_{x y}=0.33$. Entonces, todos los valores de $R_{x y}$ obtenidos son estadísticamente diferentes de cero, pero podrán mejorar al considerar $n=1$. Esto ocurre en febrero y diciembre, pero no en marzo ni en noviembre; para estos meses, se probó mejorar su relación funcional utilizando $n=2$, lo cual acontece únicamente en marzo.

\section{Coeficientes de regresión}

Definida la memoria del modelo en cada mes (tabla 6), ahora se muestran en la tabla 7, los coeficientes de regresión obtenidos mediante el ajuste de mínimos cuadrados de los residuos de la ecuación 1 (ecuaciones 2 y $3)$, con $N=35$. Los resultados de la tabla 7 permiten establecer tres tipos de modelos de regresión: (1) los de la época de estiaje (enero a mayo) con pendiente mediana de 0.038 y ordenada al origen mediana de 8.609 , similares a la figura 2; (2) los de la época de lluvias (junio a octubre) con pendiente mediana de $0.331 \mathrm{y}$ ordenada al origen mediana de 64.997, similares a la figura 3 y (3) los de la época de transición (noviembre y diciembre) con pendiente del orden de 0.073 y ordenada al origen del orden 20.

Tabla 6. Coeficientes de correlación $\left(R_{x y}\right)$ de la relación funcional en la estación hidrométrica Ballesmi para el parámetro de memoria ( $n$ ) indicado

\begin{tabular}{ccccccccccccc}
\hline Parámetro de memoria & $\mathrm{E}$ & $\mathrm{F}$ & $\mathrm{M}$ & $\mathrm{A}$ & $\mathrm{M}^{\prime}$ & $\mathrm{J}$ & $\mathrm{J}^{\prime}$ & $\mathrm{A}^{\prime}$ & $\mathrm{S}$ & $\mathrm{O}$ & $\mathrm{N}$ & $\mathrm{D}$ \\
\hline$n=0$ & 0.740 & 0.498 & 0.521 & 0.789 & 0.711 & 0.940 & 0.888 & 0.777 & 0.885 & 0.846 & 0.597 & 0.418 \\
$n=1$ & - & 0.723 & 0.599 & - & - & - & - & - & - & - & 0.559 & 0.756 \\
$n=2$ & - & - & 0.652 & - & - & - & - & - & - & - & 0.510 & - \\
\hline
\end{tabular}

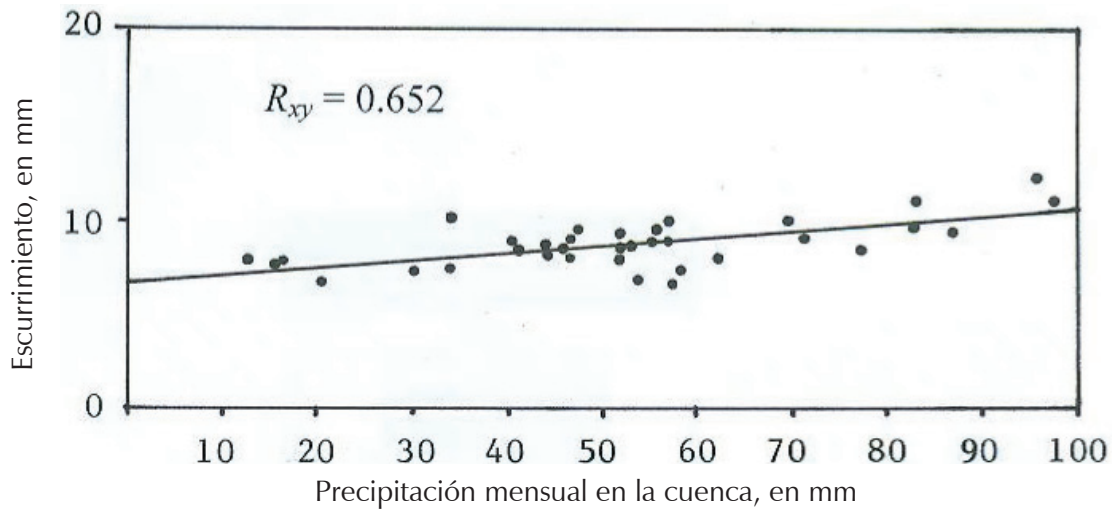

Figura 2. Relación funcional precipitación-escurrimiento del mes de marzo (con febrero y enero) en la estación hidrométrica Ballesmi 


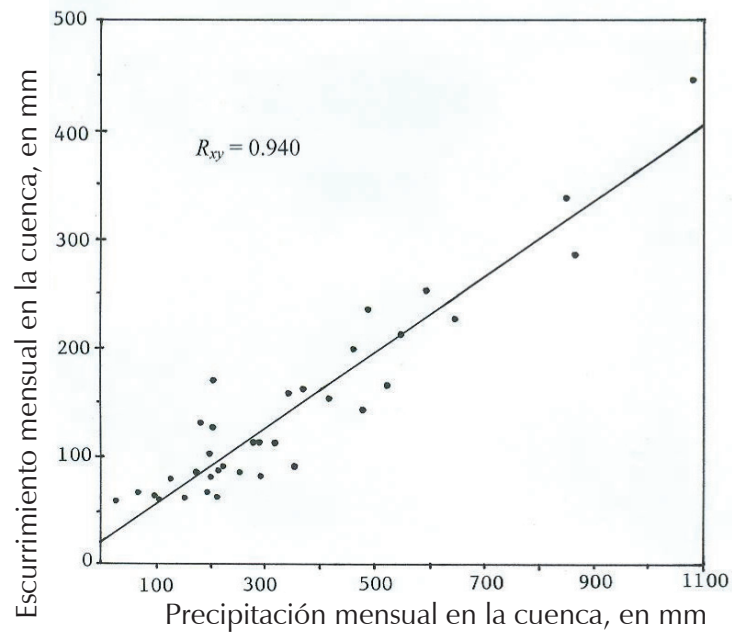

Figura 3. Relación funcional precipitación-escurrimiento del mes de junio en la estación Ballesmi

Contraste de los modelos de regresión

Contraste de medias y desviaciones estándar mensuales

Cada modelo mensual se aplica en el periodo disponible de precipitación para estimar el volumen escurrido mensual; a continuación se cuantifican las medias y desviaciones estándar mensuales, tanto de los escurrimientos estimados como de los corregidos. En la tabla 8 se han concentrado los resultados obtenidos. Se observa que existe una reproducción excelente de las medias durante todo el año; respecto a las desviaciones estándar, todos sus valores son aproximados pero inferiores, excepto en marzo. Las mayores diferencias se presentan en la época de lluvias, específicamente en agosto y noviembre.

\section{Contraste de escurrimientos anuales}

En la tabla 9 se realiza el análisis de los valores anuales, mostrando los errores relativos $(E R)$ estimados con la ecuación 7, del escurrimiento estimado con los modelos de regresión con respecto al escurrimiento corregido. Se deduce de tales resultados que los años con $E R$ extremo por exceso son 1982 y 1964 y por defecto 1990 y 1969; siendo los años 1961 y 1971 los que menor ER presentaron.

\section{Contraste de escurrimientos mensuales}

Para los seis años definidos con errores relativos extremos, en la tabla 10 se exponen sus respectivos $E R$ mensuales. Finalmente, en la figura 4 se muestra gráficamente un contraste para un lapso de ocho años, seleccionado de manera arbitraria, pero que incluyera años secos y húmedos observados (tabla 9).

Otras opciones de procesamiento

Por supuesto que el método sugerido para retirar la aportación de manantiales del escurrimiento observado en la estación de aforos Ballesmi, es aproximado y una mejor opción sería analizar con isótopos tal escurrimiento para estimar la proporción de aguas subterráneas (Custodio, 1976; Aggarwal et al., 2012).

Tabla 7. Coeficientes de regresión $\left(\beta_{j, k}\right)$ de los modelos mensuales del PPE en la estación hidrométrica Ballesmi

\begin{tabular}{|c|c|c|c|c|c|c|c|c|c|c|c|c|}
\hline $\begin{array}{l}\text { Coeficiente de } \\
\text { regresión }\end{array}$ & $\mathrm{E}$ & $\mathrm{F}$ & M & $\mathrm{A}$ & $\mathrm{M}^{\prime}$ & $\mathrm{J}$ & $\mathrm{J}^{\prime}$ & $\mathrm{A}^{\prime}$ & S & $\mathrm{O}$ & $\mathrm{N}$ & $\mathrm{D}$ \\
\hline $\begin{array}{c}\text { Ordenada al origen } \\
\left(\beta_{\mathrm{i}, 0}\right)\end{array}$ & 8.609 & 8.058 & 6.693 & 12.338 & 19.159 & 23.580 & 64.997 & 70.511 & 32.251 & 67.747 & 31.437 & 14.257 \\
\hline Pendiente $\left(\beta_{i, 1}\right)$ & 0.029 & 0.044 & 0.038 & 0.032 & 0.038 & 0.345 & 0.313 & 0.270 & 0.589 & 0.331 & 0.072 & 0.075 \\
\hline
\end{tabular}

Tabla 8. Valores observados y estimados en miles de $\mathrm{m}^{3}$ de la media aritmética $(M)$ y la desviación estándar $(D E)$ del escurrimiento mensual en la estación hidrométrica Ballesmi

\begin{tabular}{ccccccccccccc}
\hline Parámetro estadístico & $\mathrm{E}$ & $\mathrm{F}$ & $\mathrm{M}$ & $\mathrm{A}$ & $\mathrm{M}^{\prime}$ & $\mathrm{J}$ & $\mathrm{J}^{\prime}$ & $\mathrm{A}^{\prime}$ & $\mathrm{S}$ & $\mathrm{O}$ & $\mathrm{N}$ & $\mathrm{D}$ \\
\hline$M_{\text {obser }}$ & 1941 & 2007 & 1684 & 2912 & 4950 & 27540 & 29440 & 28613 & 50445 & 24015 & 7215 & 3712 \\
$M_{\text {estim }}$ & 1942 & 2008 & 1694 & 2911 & 4954 & 27526 & 29428 & 28594 & 50471 & 24028 & 7216 & 3711 \\
$D E_{\text {obser }}$ & 269 & 312 & 239 & 566 & 1086 & 17073 & 15788 & 12541 & 27406 & 8883 & 1302 & 607 \\
$D E_{\text {estim }}$ & 199 & 226 & 277 & 446 & 775 & 16047 & 14013 & 9727 & 24275 & 7525 & 778 & 458 \\
\hline
\end{tabular}


Tabla 9. Errores relativos (ER) de los volúmenes escurridos anuales estimados con los modelos de regresión mensuales de la estación de aforos Ballesmi

\begin{tabular}{|c|c|c|c|c|c|c|c|}
\hline Año & $\begin{array}{c}V E A C \\
\left(10^{3} \cdot \mathrm{m}^{3}\right)\end{array}$ & $\begin{array}{c}V E A E \\
\left(10^{3} \cdot \mathrm{m}^{3}\right)\end{array}$ & $\begin{array}{l}E R \\
(\%)\end{array}$ & Año & $\begin{array}{c}V E A C \\
\left(10^{3} \cdot \mathrm{m}^{3}\right)\end{array}$ & $\begin{array}{c}V E A E \\
\left(10^{3} \cdot \mathrm{m}^{3}\right)\end{array}$ & $\begin{array}{l}E R \\
(\%)\end{array}$ \\
\hline 1961 & 171758 & 173173 & 0.8 & 1980 & 132828 & 152376 & 14.7 \\
\hline 1962 & 120956 & 136973 & 13.2 & 1981 & 234010 & 211194 & -9.8 \\
\hline 1963 & 121217 & 139085 & 14.7 & 1982 & 94927 & 133969 & 41.1 \\
\hline 1964 & 116893 & 155906 & 33.4 & 1983 & 185609 & 196103 & 5.7 \\
\hline 1965 & 154874 & 172521 & 11.4 & 1984 & 259231 & 234859 & -9.4 \\
\hline 1966 & 201977 & 191634 & -5.1 & 1985 & 166369 & 173400 & 4.2 \\
\hline 1967 & 222492 & 212351 & -4.6 & 1986 & 158084 & 169029 & 6.9 \\
\hline 1968 & 186383 & 193207 & 3.7 & 1987 & 157676 & 146350 & -7.2 \\
\hline 1969 & 221437 & 185397 & -16.3 & 1988 & 204658 & 172103 & -15.9 \\
\hline 1970 & 227033 & 236284 & 4.1 & 1989 & 151575 & 158670 & 4.7 \\
\hline 1971 & 201357 & 201061 & -0.1 & 1990 & 197155 & 156199 & -20.8 \\
\hline 1972 & 196558 & 195126 & -0.7 & 1992 & 196947 & 187835 & -4.6 \\
\hline 1973 & 236493 & 222008 & -6.1 & 1993 & 321590 & 271427 & -15.6 \\
\hline 1974 & 228230 & 221820 & -2.8 & 1994 & 152815 & 177375 & 16.1 \\
\hline 1975 & 246746 & 231249 & -6.3 & 1995 & 146017 & 157199 & 7.7 \\
\hline 1976 & 248487 & 241946 & -2.6 & 1996 & 164994 & 175998 & 6.7 \\
\hline 1977 & 115343 & 136009 & 17.9 & 1977 & 116054 & 130309 & 12.3 \\
\hline 1978 & 197815 & 206770 & 4.5 & Prom. & 184474 & 184483 & - \\
\hline
\end{tabular}

VEAC volumen escurrido anual corregido

VEAE volumen escurrido anual estimado

Tabla 10. Errores relativos (ER) de los volúmenes escurridos mensuales estimados con los modelos de regresión respectivos de la estación de aforos Ballesmi, para los años con ER extremos

\begin{tabular}{|c|c|c|c|c|c|c|c|c|c|c|c|c|c|c|}
\hline $\begin{array}{c}\text { Año } \\
\text { (ER anual) }\end{array}$ & $\begin{array}{l}\text { Vol. Esc. } \\
\left(10^{3} \cdot \mathrm{m}^{3}\right)\end{array}$ & E & $\mathrm{F}$ & M & A & $\mathrm{M}^{\prime}$ & $\mathrm{J}$ & $\mathrm{J}^{\prime}$ & $\mathrm{A}^{\prime}$ & S & $\mathrm{O}$ & $\mathrm{N}$ & $\mathrm{D}$ & Anual \\
\hline 1982 (41.1\%) & corregido & 1760 & 1720 & 1531 & 2849 & 5730 & 11442 & 13023 & 12636 & 21325 & 14903 & 5023 & 2985 & 94927 \\
\hline 1982 (41.1 \%) & & 1787 & 1916 & 1711 & 3212 & 5325 & 6335 & 15964 & 19251 & 40942 & 27825 & 6383 & 3318 & 133969 \\
\hline $1982(41.1 \%)$ & $E R(\%)$ & 1.5 & 11.4 & 11.8 & 12.7 & -7.1 & -44.6 & 22.6 & 52.4 & 92.0 & 86.7 & 27.1 & 11.2 & $92.0(-44.6)$ \\
\hline $1964(33.4 \%)$ & corregido & 1659 & 1713 & 1403 & 2757 & 4675 & 17819 & 13991 & 12669 & 28897 & 18490 & 7337 & 5483 & 116895 \\
\hline $1964(33.4 \%)$ & estimado & 1747 & 1651 & 1811 & 2519 & 5137 & 19348 & 17685 & 18630 & 56885 & 16448 & 9388 & 4655 & 155906 \\
\hline $1964(33.4 \%)$ & $E R(\%)$ & 5.3 & -3.6 & 29.1 & -8.6 & 9.9 & 8.6 & 26.4 & 47.1 & 96.9 & -11.0 & 28.0 & -15.1 & $96.9(-15.1)$ \\
\hline $1990(-20.8 \%)$ & corregido & 2122 & 1794 & 1716 & 3751 & 4237 & 11020 & 25269 & 62807 & 46178 & 25875 & 7602 & 4178 & 197155 \\
\hline $1990(-20.8 \%)$ & estimado & 2074 & 1994 & 1797 & 2987 & 4591 & 11664 & 25219 & 34378 & 32469 & 27930 & 7353 & 3742 & 156199 \\
\hline $1990(-20.8 \%)$ & $E R(\%)$ & -2.3 & 11.1 & 4.7 & -20.4 & 8.4 & 0.3 & -0.2 & -45.3 & -29.7 & 7.9 & -3.3 & -10.4 & $11.1(-45.3)$ \\
\hline $1969(-16.3 \%)$ & corregido & 2100 & 2164 & 1913 & 2859 & 4771 & 13370 & 26874 & 40254 & 92327 & 24124 & 6962 & 3719 & 221436 \\
\hline $1969(-16.3 \%)$ & estimado & 1866 & 1900 & 1469 & 2798 & 4267 & 17619 & 18376 & 47716 & 53500 & 25716 & 6839 & 3331 & 185397 \\
\hline $1969(-16.3 \%)$ & $E R(\%)$ & -11.1 & -12.2 & -23.2 & -2.1 & -10.6 & 31.8 & -31.6 & 18.5 & -42.1 & 6.6 & -1.8 & -10.4 & $31.8(-45.3)$ \\
\hline $1961(0.8 \%)$ & corregido & 2141 & 2093 & 1672 & 2490 & 3905 & 31778 & 37334 & 29756 & 32995 & 15795 & 8180 & 3619 & 171759 \\
\hline $1961(0.8 \%)$ & & 2210 & 2123 & 1501 & 2451 & 3989 & 39454 & 35084 & 23487 & 35859 & 15826 & 7523 & 3665 & 173173 \\
\hline $1961(0.8 \%)$ & $E R(\%)$ & 3.2 & 1.4 & -10.2 & -1.6 & 2.2 & 24.2 & -6.0 & -21.1 & 8.7 & 0.2 & -8.0 & 1.3 & $24.2(-21.1)$ \\
\hline $1971(-0.1 \%)$ & corregido & 1898 & 1826 & 1488 & 2808 & 4487 & 29384 & 17678 & 37000 & 54192 & 39339 & 7270 & 3987 & 201358 \\
\hline $1971(-0.1 \%)$ & estimado & 1760 & 1664 & 1481 & 2539 & 5830 & 32297 & 18406 & 32286 & 64888 & 29197 & 6866 & 3848 & 201061 \\
\hline $1971(-0.1 \%)$ & $E R(\%)$ & -7.3 & -8.9 & -0.5 & -9.6 & 29.9 & 9.9 & 4.1 & -12.7 & 19.7 & -25.8 & -5.6 & -3.5 & $29.9(-25.8)$ \\
\hline
\end{tabular}



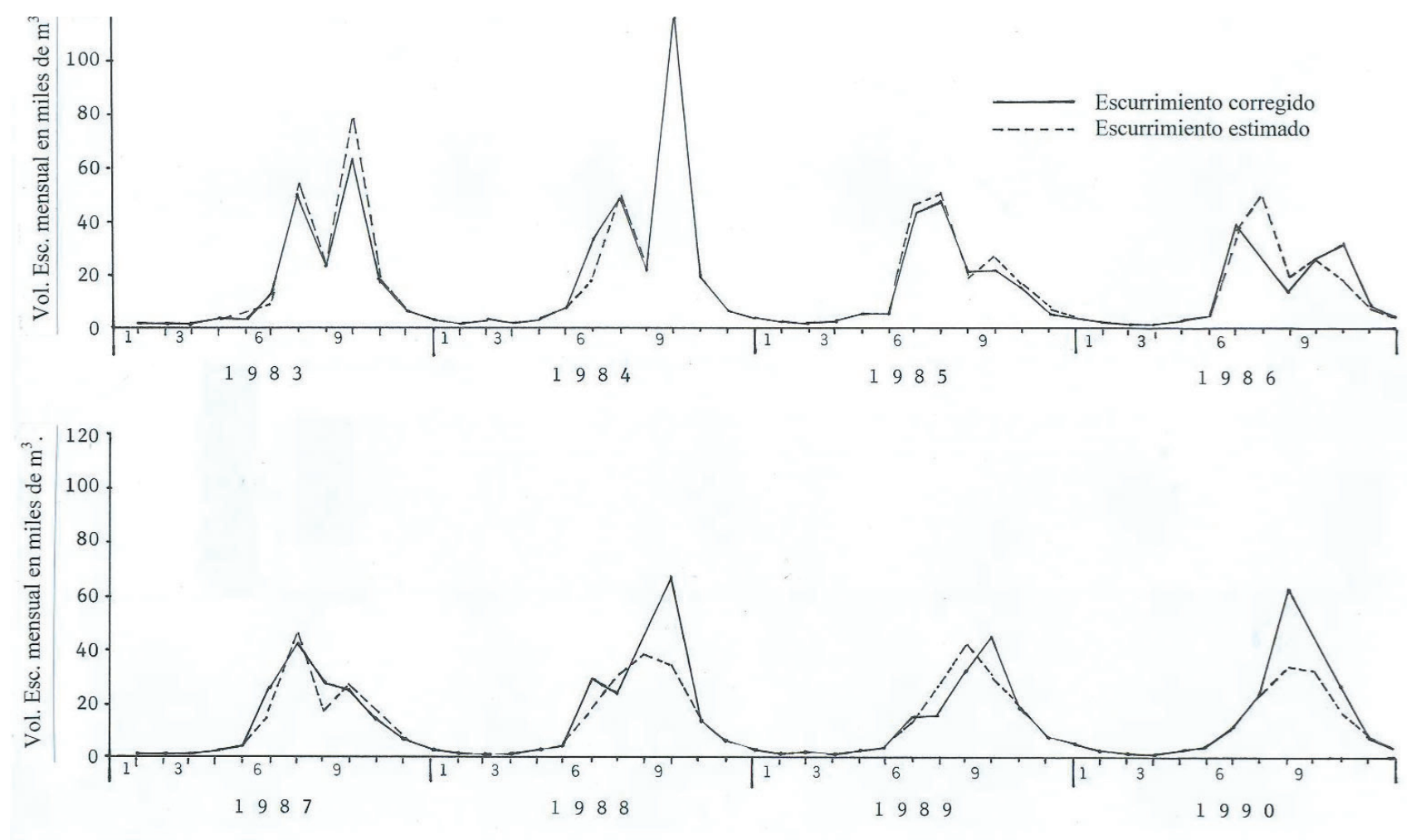

Figura 4. Contraste de volúmenes escurridos mensuales corregidos y estimados con los modelos de regresión en la estación hidrométrica Ballesmi para los años indicados

Por otra parte, se consideró un mejor planteamiento de análisis, el procesamiento de todo el registro conjunto y su contraste; sin embargo, otra opción consiste en dividir tal registro y calibrar con una mitad y contrastar en la otra. Este último enfoque es el adoptado en la aplicación de la mayoría de los modelos hidrológicos conceptuales. En este trabajo los objetivos centrales fueron la corrección de un registro por aportación de manantiales y la verificación general del modelo más simple del proceso precipitación-escurrimiento mensual.

\section{Conclusiones}

Primera: Al observarse una similitud numérica en los coeficientes de escurrimiento promedio mensuales en cuencas que pertenecen a la misma subregión geográfica (Campos, 2013), el procedimiento sugerido para eliminar del volumen escurrido mensual observado la aportación subterránea estimada, se puede considerar aproximado.

Segunda: el modelo matemático más simple del proceso precipitación-escurrimiento (PPE) mensual que se pue- de establecer es la regresión lineal o curva, la cual, debido a su planteamiento conceptual, reproduce fielmente los volúmenes escurridos promedio mensual y alcanza una excelente aproximación en relación con su dispersión, como se ha demostrado en la tabla 8 , con base en las medias y desviaciones estándar.

Tercera: a nivel anual, el contraste entre los escurrimientos observados corregidos y los estimados con los modelos de regresión, según resultados concentrados en la tabla 9, indica que se reproducen sus magnitudes, así como su variabilidad.

Cuarta: finalmente, a nivel mensual y de acuerdo a los indicadores extremos mostrados en la tabla $10 \mathrm{y}$ la figura 4 que muestra un lapso del registro modelado, se considera que la reproducción del escurrimiento es bastante aproximada. Debido a ello, se recomienda la aplicación de este tipo de modelos del PPE, para deducir datos faltantes y ampliar registros de escurrimiento en cuencas de climas húmedos que tienen información de precipitación de mayor amplitud. 


\section{Agradecimiento}

Se agradece al Ing. Armando Rocha Hernández, Jefe del Centro de Previsión Meteorológica de la Dirección Estatal San Luis Potosí de la CONAGUA, proporcionar al autor toda la información pluviométrica procesada.

\section{Referencias}

Aggarwal P.K., Fröhlich K.O., Gat J.R., Gonfiantini R. Isotope Hydrology, Benchmark Papers in Hydrology \#8, International Association of Hydrological Sciences Press. CEH Wallingford, Oxfordshire, Reino Unido, 2012.

Beven K.J. Rainfall-Runoff Modelling. The Primer, Capítulo 1, Down to Basics: Runoff Processes and the Modelling Process, John Wiley \& Sons, Ltd. Chichester, Inglaterra, 2001, pp. 1-23.

Buishand T.A. Some Methods for Testing the Homogeneity of Rainfall Records. Journal of Hydrology, volumen 58, 1982: 11-27.

Campos-Aranda D.F. Introducción a los métodos numéricos: Software en Basic y aplicaciones en hidrología superficial, capítulo 5, Ajuste de curvas, Editorial Universitaria Potosina, San Luis Potosí, México, 2003, pp. 93-127.

Campos-Aranda D.F. Agroclimatología cuantitativa de cultivos, capítulo 4, análisis de la precipitación, y anexo B, FDP Gamma Mixta, Editorial Trillas, México, 2005, pp. 97-120 y 267-281.

Campos-Aranda D.F. Coeficientes de escurrimiento promedio mensuales: comportamiento y aplicación en la estimación de la disponibilidad. Revista digital Tláloc, edición 61, octubrediciembre de 2013, 23 p.

Custodio E. Trazadores y técnicas radioisotópicas en hidrología subterránea, sección 12, pp. 1165-1312 en: Hidrología subterránea, tomo II, Directores de Edición: Emilio Custodio y Manuel Ramón Llamas, ediciones Omega, Barcelona, España, 1976, 1194 p.

Haan C.T., Johnson H.P., Brakensiek D.L. Hydrologic Modeling of Small Watersheds, ASAE Monograph Núm. 5, St. Joseph, Michigan, EUA, 1982, 533 p.
IMTA (Instituto Mexicano de Tecnología del Agua). Banco Nacional de Datos de Aguas Superficiales (BANDAS), 8 CD's, CONAGUA-SEMARNAT-IMTA, Jiutepec, Morelos, 2002.

INEGI (Instituto Nacional de Estadística, Geografía e Informática). Carta de Precipitación Total Anual: México, Aguascalientes, México, 1980.

Jones J.A.A. Global Hydrology: Processes, Resources and Environmental Management, capítulo 6, Modelling Runoff Processes, Pearson Education, Harlow, Inglaterra, 1997, pp. 171-210.

Mimikou M. y Rao R. Regional Monthly Rainfall-Runoff Model. Journal of Water Resources Planning and Management, volumen 109 (número 1), enero de 1983: 75-93.

Shaw E.M., Beven K.J., Chapell N.A., Lamb R. Hydrology in Practice, 4 a ed., capítulo 12, Catchment Modelling, Spon Press, Londres, Inglaterra, 2011, pp. 280-321.

Singh V.P. Computer Models of Watershed Hydrology, capítulo 1, Watershed Modeling, Water Resources Publications, Highlands Ranch, Colorado, EUA, 1995, pp. 1-22.

WMO (World Meteorological Organization). Climatic Change, Annexed III: Standard Tests of Significance to be Recommended in Routine Analysis of Climatic Fluctuations, pp. 58-71. Technical Note No. 79, WMO-No. 195. Secretariat of the WMO, Génova, Suiza, 1971, 79 p.

Yevjevich V. Probability and Statistics in Hydrology, capítulo 11, Correlation and Regression, Water Resources Publications, Fort Collins, Colorado, EUA, 1972, pp. 232-276.

Este artículo se cita:
Citación estilo Chicago
Campos-Aranda, Daniel Francisco. Modelación del proceso preci-
pitación-escurrimiento mensual por medio de regresiones. Inge-
niería Investigación y Tecnología, XV, 04 (2014): 625-636.
Citación estilo ISO 690
Campos-Aranda D.F. Modelación del proceso precipitación-escu-
rrimiento mensual por medio de regresiones. Ingeniería Investiga-
ción y Tecnología, volumen XV (número 4), octubre-diciembre
2014: 625-636.

\footnotetext{
Semblanza del autor

Daniel Francisco Campos-Aranda. Obtuvo el título de ingeniero Civil en diciembre de 1972, en la entonces Escuela de Ingeniería de la UASLP. Durante el primer semestre de 1977, realizó en Madrid, España un diplomado en hidrología general y aplicada. Posteriormente, durante 1980-1981 llevó a cabo estudios de maestría en ingeniería en la especialidad de Hidráulica, en la División de Estudios de Posgrado de la Facultad de Ingeniería de la UNAM. En esta misma institución, inició (1984) y concluyó (1987) el doctorado en ingeniería con especialidad en aprovechamientos hidráulicos. Ha publicado artículos principalmente en revistas mexicanas de excelencia: 42 en Tecnología y Ciencias del Agua (antes Ingeniería Hidráulica en México), 17 en Agrociencia y 14 en Ingeniería. Investigación y Tecnología. Es profesor jubilado de la UASLP, desde el $1^{\circ}$ de febrero de 2003. En noviembre de 1989 obtuvo la medalla Gabino Barreda de la UNAM y en 2008 le fue otorgado el Premio Nacional "Francisco Torres H." de la AMH. A partir de septiembre de 2013 vuelve a ser investigador nacional nivel I.
} 